shows that cellulose and its derivatives have scope for analysis and separations, now that a wide variety of these products are available with well defined properties.

The next three articles come under the heading of gas chromatography. This form, more than any other, depends on the automatic recording of the issuing components by the detector. Manual interpretation of the plots for identification and quantitation can be timeconsuming. The author of the first article $(\mathrm{H}$. Wilson Johnson, jun.) believes that it is possible to achieve this stage automatically. He discusses in detail the technical problems involved and his article should help to hasten the time when the gas chromatogram becomes completely automatic from start to finish. The next article is an up to date survey by A. P. Altshuler of atmospheric analysis by gas chromatography. It includes developments in detectors of such high sensitivity that concentration of trace quantities of pollutants from large amounts of air are unnecessary. Atmospheric pollution analysis has its own special problems and the technical developments which should overcome them are discussed. The final article, by J. D. Wineforder and T. H. Glenn, covers non-ionization detectors. In general these are less sensitive than ionization detectors, but are more selective and hence of advantage for identification. The many different types of non-ionization detector are discussed, including those of very recent development.

Like its predecessors this volume contains something of interest for all who use chromatographic methods. It continues the artificial division of chromatography into "General" and "Gas". If the latter is so important, does it not deserve a separate "Advances"? R. ConsDen

\section{EUCLIDEAN AND NON-EUCLIDEAN}

Foundation of Euclidean and Non-Euclidian Geometries According to $\mathrm{F}$. Klein

By L. Rédei. (International Series of Monographs in Pure and Applied Mathematics, Vol. 97.) Pp. $x+400$. (Pergamon: Oxford, London and New York, 1968.) $150 s$.

For some two thousand years after Euclid, mathematicians tried to prove- on the basis of the other axiomsthe Parallel Postulate, which may be stated in the following form: given a line in a plane and a point not on the line, there exists a unique line in the plane passing through the point and parallel to the given line. It was Gauss who first showed the impossibility of the task by constructing a consistent geometry in which the postulate is false but the other axioms remain truo. Two such non-Euclidean geometries exist: hyperbolic geometry (due to Lobachevski and Bolyai), in which there are at least two lines through the point parallel to the given line, and elliptic geometry (due to Riemann), in which there is no line parallel to the given line.

Klein's "Erlangen Programm" of 1872 suggested that a geometry is the theory of the properties of a set invariant under a given group of permutations of the set. Professor Rédei has written a unified axiomatic treatment of threedimensional elliptic, hyperbolic and parabolic (Euclidean) real geometry. The axioms are separated into four typesaxioms of incidence, betweenness, continuity and motion. The entire book then consists of deriving the basic properties of the geometry obtained by successively adding the different types of axioms. First, the projective closure $\bar{\Omega}$ of the affine geometry $\Omega$ realized from the axioms of incidence is considered; then, the group of all collineations of $\bar{\Omega}$. The three geometries aimed at all have groups which are sub-groups of the collineation group and are thus sub-geometrics of that of $\bar{\Re}$ in Klein's sense.
The theorem which finally establishes the three different geometries depends on the improper points (those points of $\bar{\Omega}$ not in $\Omega$ ) and the proper lines (those lines of $\bar{\Omega}$ which contain at least one proper point). It is shown that there aro three possibilities: (i) there exists no improper point; (ii) every proper line contains just one improper point; (iii) every proper line contains an infinity of improper points.

These cases give rise respectively to elliptic, parabolic and hyperbolic geometry. The consistoncy of each geometry is demonstrated by an analytic model.

The book provides a complete account of the foundations of the subject, so much so that there is little room at the end for further development. It is fundamental but not elementary and is not recommended as an introduction to the subject. It will be, however, a basic reference which, despite the generous number of misprints, every library should possoss and, indeed, which only a library could afford.

James Hursohfexd

\section{PHARMACOLOGY FOR STUDENTS}

Pharmacological Principles and Practice

By J. P. Payne and W. D. M. Paton. Pp. ix +417. (Churchill: London, 1968.) $75 s$.

Most teachers of pharmacology of experience will probably agree on certain demands they would like to make on a new textbook of pharmacology for medical students(1) It should be short, because at whatever period of the curriculum the student is introduced to pharmacology, the demands of all subjects are steadily increasing. (2) It must not be overloaded with the names of drugs in the same category and with the same principal action. The number of drugs has so much increased in the past decades that the beginner will even have diffieulties to memorize the names of those compounds which have been chosen to represent a given group of drugs. (Quoting a large number of closely related drugs is, of course, necessary when structure-action relationships are to be discussed, but once these relationships are understood there is no need to memorize the whole series.) (3) Recent advances in biochemical pharmacology must be duly considered. In most medical schools the student has a better basis of biochemistry than he had only five years ago and the pharmacologist not only can but should build up on that to indicate that we are approaching an era in which not only the "pharmacology of organs" but also the cellular action of drugs is beginning to be understood. (4) Pharmacological concepts are frequently so closely intermingled with physiological ones that some overlap with textbooks of physiology is unavoidable. This can be reduced to the right measure if the approach is integrative rathor than descriptive. (5) The student is usually at a stage of his studies when he is longing to apply himself to clinical problems. A textbook for medical students should therefore illustrate analyses of drug actions by references to clinical experiences whenever possible.

The book fulfils all these criteria. It is a clear and comprehensive account of the subject. There is a good selection of papers and review articles appended to each chapter, if the student wishes to read further. Its first chapter is a masterly account of the principles of general pharmacology and one marvels that the authors were able to put so much into so few pages. It might be objected that the accounts of the action of individual drugs which follow are too dogmatic but this is a "good" fault. Nondogmatic statements could only be introduced at the price of explaining controversial drugs more fully, otherwise the student will be confused or will appear to be critical without understanding the reasons for his criticism. 